\title{
Monotone iterative method for differential systems with coupled integral boundary value problems
}

Yujun Cui ${ }^{1,2^{*}}$ and Yumei Zou ${ }^{3}$

\section{"Correspondence:}

cyj720201@163.com

${ }^{1}$ School of Mathematical Sciences,

Qufu Normal University, Qufu,

Shandong 273165, P.R. China

${ }^{2}$ Department of Mathematics,

Shandong University of Science and

Technology, Qingdao, 266590,

P.R. China

Full list of author information is

available at the end of the article

\begin{abstract}
By establishing a comparison result and using the method of upper and lower solutions and the monotone iterative technique, we investigate the differential systems with coupled integral boundary value problems. Sufficient conditions are established for the existence of an extremal system of solutions of the given problem.
\end{abstract} MSC: 34B15

Keywords: monotone iterative technique; upper and lower solutions; coupled integral boundary conditions; Fredholm theorem

\section{Introduction}

We will devote the paper to considering the existence of a solution of coupled integral boundary value conditions for the second-order ordinary differential system (ODS for short)

$$
\left\{\begin{array}{l}
-x^{\prime \prime}(t)=f_{1}(t, x(t), y(t)), \quad t \in(0,1), \\
-y^{\prime \prime}(t)=f_{2}(t, x(t), y(t)), \quad t \in(0,1), \\
x(0)=y(0)=0, \quad x(1)=\int_{0}^{1} y(t) d A(t), \quad y(1)=\int_{0}^{1} x(t) d B(t),
\end{array}\right.
$$

where $A$ and $B$ are right continuous on $[0,1)$, left continuous at $t=1$ and nondecreasing on $[0,1], A(0)=B(0)=0 ; \int_{0}^{1} u(s) d A(s)$ and $\int_{0}^{1} u(s) d B(s)$ denote the Riemann-Stieltjes integrals of $u$ with respect to $A$ and $B$, respectively.

The theory of differential system with coupled boundary value conditions is an important branch of nonlinear analysis. It is worth mentioning that a differential system with coupled boundary value conditions appears often in investigations connected with mathematical physics, mathematical biology, biochemical system and so on (see [1-3]). One of the basic problems considered in the theory of differential system with coupled boundary value conditions is to establish convenient conditions guaranteeing the existence of solutions of those equations. However, the theory of coupled boundary value problems for a differential system is still in the initial stages.

The monotone iterative technique combined with the method of upper and lower solutions is a powerful tool for proving the existence of solutions of differential equations/system (see [4-9] and the references therein), the advantage and importance of the technique needs no special emphasis [10-12]. The basic idea of this method is that us-

C2013 Cui and Zou; licensee Springer. This is an Open Access article distributed under the terms of the Creative Commons Attribution License (http://creativecommons.org/licenses/by/2.0), which permits unrestricted use, distribution, and reproduction in any medium, provided the original work is properly cited. 
ing the upper and lower solutions as an initial iteration, one can construct monotone sequences from the corresponding linear differential equations/system, and these sequences converge monotonically to the maximal and minimal solutions of the nonlinear differential equations/system. When the method is applied to a differential system with coupled boundary value conditions, it usually needs suitable differential inequalities as a comparison principle. The results in this paper are inspired by [13]. Here, we establish differential inequalities as a comparison principle, i.e., Lemma 2.2. Then, we give a different proof for the existence and uniqueness of the solutions for linear coupled boundary value conditions for a differential system, i.e., Lemma 2.5. Finally, by use of the monotone iterative technique and the method of upper and lower solutions, we obtain the existence result of extremal solutions for (1.1).

\section{Preliminary results}

Let

$$
\kappa_{1}=\int_{0}^{1} t d A(t), \quad \kappa_{2}=\int_{0}^{1} t d B(t), \quad \kappa=1-\kappa_{1} \kappa_{2}, \quad E=C^{2}[0,1] .
$$

We make assumptions involving $\kappa_{1}, \kappa_{2}$ and $\kappa$ as follows.

$\left(\mathrm{H}_{1}\right) \kappa_{1}>0, \kappa_{2}>0, \kappa>0$.

Definition 2.1 $\left(u_{0}, v_{0}\right) \in E \times E$ is called a lower system of solutions of differential system (1.1) if

$$
\begin{cases}-u_{0}^{\prime \prime}(t) \leq f_{1}\left(t, u_{0}(t), v_{0}(t)\right), & t \in(0,1), \\ -v_{0}^{\prime \prime}(t) \leq f_{2}\left(t, u_{0}(t), v_{0}(t)\right), & t \in(0,1), \\ u_{0}(0) \leq 0, \quad v_{0}(0) \leq 0, & u_{0}(1) \leq \int_{0}^{1} v_{0}(t) d A(t), \quad v_{0}(1) \leq \int_{0}^{1} u_{0}(t) d B(t) .\end{cases}
$$

Analogously, $\left(\alpha_{0}, \beta_{0}\right) \in E \times E$ is called an upper system of solutions of differential system (1.1) if

$$
\begin{cases}-\alpha_{0}^{\prime \prime}(t) \geq f_{1}\left(t, \alpha_{0}(t), \beta_{0}(t)\right), \quad t \in(0,1), & \\ -\beta_{0}^{\prime \prime}(t) \geq f_{2}\left(t, \alpha_{0}(t), \beta_{0}(t)\right), \quad t \in(0,1), & \\ \alpha_{0}(0) \geq 0, \quad \beta_{0}(0) \geq 0, \quad \alpha_{0}(1) \geq \int_{0}^{1} \beta_{0}(t) d A(t), \quad \beta_{0}(1) \geq \int_{0}^{1} \alpha_{0}(t) d B(t) .\end{cases}
$$

In what follows, we assume that

$$
u_{0}(t) \leq \alpha_{0}(t), \quad v_{0}(t) \leq \beta_{0}(t), \quad t \in[0,1]
$$

and define that sector

$$
\Omega=\left\{(\xi, \eta) \in E \times E:\left(u_{0}(t), v_{0}(t)\right) \leq(\xi(t), \eta(t)) \leq\left(\alpha_{0}(t), \beta_{0}(t)\right), t \in[0,1]\right\},
$$

where the vectorial inequalities mean that the same inequalities hold between their corresponding components.

We present new comparison results and lemmas which are crucial for our discussion. 
Lemma 2.1 [11, 14] Suppose that $-\infty<a<b<+\infty$. If there exist $M \in C\left[[a, b], R^{+}\right]$and $p \in C^{2}[a, b]$ satisfying

$$
\left\{\begin{array}{l}
-p^{\prime \prime}(t) \leq-M(t) p(t), \quad t \in(0,1) \\
p(a) \leq 0, \quad p(b) \leq 0
\end{array}\right.
$$

then $p(t) \leq 0, t \in[a, b]$.

Lemma 2.2 Suppose that $\left(\mathrm{H}_{1}\right)$ holds. Let $x, y \in E$ satisfy

$$
\left\{\begin{array}{l}
-x^{\prime \prime}(t) \leq-M(t) x(t), \quad-y^{\prime \prime}(t) \leq-N(t) y(t), \quad t \in(0,1) \\
x(0) \leq 0, y(0) \leq 0, \quad x(1) \leq \int_{0}^{1} y(t) d A(t), \quad y(1) \leq \int_{0}^{1} x(t) d B(t),
\end{array}\right.
$$

where $M(t), N(t) \in C\left[[0,1], R^{+}\right]$. Then $x(t) \leq 0, y(t) \leq 0, t \in[0,1]$.

Proof Suppose the contrary. By Lemma 2.1, one easily sees that there are only three cases to consider:

Case 1. $x(1) \leq 0$ and $y(1)>0$. By Lemma 2.1, $x(t) \leq 0$ for all $t \in[0,1]$. Then $y(1) \leq$ $\int_{0}^{1} x(t) d B(t) \leq 0$, which contradicts $y(1)>0$.

Case 2. $y(1) \leq 0$ and $x(1)>0$. By Lemma 2.1, $y(t) \leq 0$ for all $t \in[0,1]$. So, we have $x(1) \leq$ $\int_{0}^{1} y(t) d B(t) \leq 0$, which contradicts $x(1)>0$.

Case 3. $x(1)>0$ and $y(1)>0$. There are $\xi, \eta \in[0,1)$ such that

$$
x(t) \leq 0, \quad t \in[0, \xi], \quad x(t) \geq 0, \quad t \in[\xi, 1],
$$

and

$$
y(t) \leq 0, \quad t \in[0, \eta], \quad y(t) \geq 0, \quad t \in[\eta, 1] .
$$

It follows from (2.2) that

$$
-x^{\prime \prime}(t) \leq 0, \quad t \in[\xi, 1] \quad \text { and } \quad-y^{\prime \prime}(t) \leq 0, \quad t \in[\eta, 1] .
$$

Hence, we have

$$
x(t) \leq x(\xi) \frac{1-t}{1-\xi}+x(1) \frac{t-\xi}{1-\xi}=x(1) \frac{t-\xi}{1-\xi}, \quad t \in[\xi, 1],
$$

and

$$
y(t) \leq y(\eta) \frac{1-t}{1-\eta}+y(1) \frac{t-\eta}{1-\eta}=y(1) \frac{t-\eta}{1-\eta}, \quad t \in[\eta, 1] .
$$

Therefore,

$$
\begin{aligned}
x(1) & \leq \int_{0}^{\eta} y(t) d A(t)+\int_{\eta}^{1} y(t) d A(t) \leq \int_{\eta}^{1} y(t) d A(t) \\
& \leq y(1) \int_{\eta}^{1} \frac{t-\eta}{1-\eta} d A(t) \leq y(1) \int_{0}^{1} t d A(t)=\kappa_{1} y(1),
\end{aligned}
$$


and

$$
\begin{aligned}
y(1) & \leq \int_{0}^{\xi} x(t) d B(t)+\int_{\xi}^{1} x(t) d B(t) \leq \int_{\xi}^{1} x(t) d B(t) \\
& \leq x(1) \int_{\xi}^{1} \frac{t-\xi}{1-\xi} d B(t) \leq x(1) \int_{0}^{1} t d B(t)=\kappa_{2} x(1) .
\end{aligned}
$$

The last two inequalities give

$$
0<x(1) \leq \kappa_{1} y(1) \leq \kappa_{1} \kappa_{2} x(1),
$$

which implies that $1 \leq \kappa_{1} \kappa_{2}$, a contradiction. Hence, $x(t) \leq 0, y(t) \leq 0, t \in[0,1]$.

Lemma 2.3 Suppose that $\left(\mathrm{H}_{1}\right)$ holds. Let $x, y \in E$ satisfy

$$
\left\{\begin{array}{lr}
-x^{\prime \prime}(t)=-M(t) x(t), & -y^{\prime \prime}(t)=-N(t) y(t), \quad t \in(0,1), \\
x(0)=0, \quad y(0)=0, & x(1)=\int_{0}^{1} y(t) d A(t), \quad y(1)=\int_{0}^{1} x(t) d B(t),
\end{array}\right.
$$

where $M(t), N(t) \in C\left[[0,1], R^{+}\right]$. Then $x(t)=y(t)=0, t \in[0,1]$.

The proof of Lemma 2.3 is easy, so we omit it.

Consider the differential system of BVPs

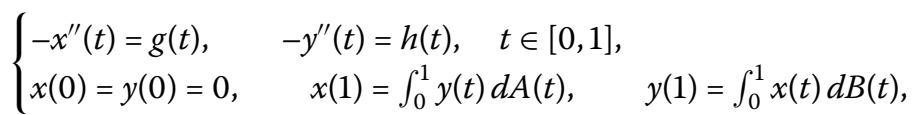

where $g, h \in C[0,1]$.

Lemma 2.4 Assume that $\left(\mathrm{H}_{1}\right)$ holds. Then $(x, y) \in E \times E$ is a system of solutions of BVPs (2.3) if and only if $(x, y) \in C[0,1] \times C[0,1]$ is a system of solutions of the integral equation

$$
\left\{\begin{array}{l}
x(t)=\int_{0}^{1} G_{1}(t, s) g(s) d s+\int_{0}^{1} H_{1}(t, s) h(s) d s, \\
y(t)=\int_{0}^{1} G_{2}(t, s) h(s) d s+\int_{0}^{1} H_{2}(t, s) g(s) d s
\end{array}\right.
$$

where

$$
\begin{array}{ll}
G_{1}(t, s)=\frac{\kappa_{1} t}{\kappa} \int_{0}^{1} k(s, \tau) d B(\tau)+k(t, s), & H_{1}(t, s)=\frac{t}{\kappa} \int_{0}^{1} k(s, \tau) d A(\tau), \\
G_{2}(t, s)=\frac{\kappa_{2} t}{\kappa} \int_{0}^{1} k(s, \tau) d A(\tau)+k(t, s), & H_{2}(t, s)=\frac{t}{\kappa} \int_{0}^{1} k(s, \tau) d B(\tau), \\
k(t, s)= \begin{cases}t(1-s), & 0 \leq t \leq s \leq 1, \\
s(1-t), & 0 \leq s \leq t \leq 1 .\end{cases}
\end{array}
$$

Proof First, suppose that $(x, y) \in E \times E$ is a system of solutions of BVPs (2.3). It is easy to see that (2.3) is equivalent to the system of integral equations

$$
x(t)=x(1) t+\int_{0}^{1} k(t, s) g(s) d s, \quad t \in[0,1]
$$




$$
y(t)=y(1) t+\int_{0}^{1} k(t, s) h(s) d s, \quad t \in[0,1] .
$$

Integrating (2.5) and (2.6) with respect to $d B(t)$ and $d A(t)$ respectively on $[0,1]$ gives

$$
\begin{aligned}
& \int_{0}^{1} x(t) d B(t)=x(1) \int_{0}^{1} t d B(t)+\int_{0}^{1} \int_{0}^{1} k(t, s) g(s) d s d B(t), \\
& \int_{0}^{1} y(t) d A(t)=y(1) \int_{0}^{1} t d A(t)+\int_{0}^{1} \int_{0}^{1} k(t, s) h(s) d s d A(t) .
\end{aligned}
$$

Therefore,

$$
\left(\begin{array}{cc}
-\kappa_{2} & 1 \\
1 & -\kappa_{1}
\end{array}\right)\left(\begin{array}{l}
x(1) \\
y(1)
\end{array}\right)=\left(\begin{array}{l}
\int_{0}^{1} \int_{0}^{1} k(t, s) g(s) d s d B(t) \\
\int_{0}^{1} \int_{0}^{1} k(t, s) h(s) d s d A(t)
\end{array}\right)
$$

and so

$$
\left(\begin{array}{l}
x(1) \\
y(1)
\end{array}\right)=\frac{1}{\kappa}\left(\begin{array}{cc}
\kappa_{1} & 1 \\
1 & \kappa_{2}
\end{array}\right)\left(\begin{array}{l}
\int_{0}^{1} \int_{0}^{1} k(t, s) g(s) d s d B(t) \\
\int_{0}^{1} \int_{0}^{1} k(t, s) h(s) d s d A(t)
\end{array}\right) .
$$

Substituting (2.7) into (2.5) and (2.6), we have

$$
\begin{aligned}
x(t)= & \frac{\kappa_{1} t}{\kappa} \int_{0}^{1} \int_{0}^{1} k(t, s) g(s) d s d B(t)+\frac{t}{\kappa} \int_{0}^{1} \int_{0}^{1} k(t, s) h(s) d s d A(t) \\
& +\int_{0}^{1} k(t, s) g(s) d s, \\
y(t)= & \frac{t}{\kappa} \int_{0}^{1} \int_{0}^{1} k(t, s) g(s) d s d B(t)+\frac{\kappa_{2} t}{\kappa} \int_{0}^{1} \int_{0}^{1} k(t, s) h(s) d s d A(t) \\
& +\int_{0}^{1} k(t, s) h(s) d s,
\end{aligned}
$$

which is equivalent to system (2.4).

Conversely, assume that $(x, y) \in C[0,1] \times C[0,1]$ is a system of solutions of an integral equation. Direct differentiation on (2.4) implies

$$
-x^{\prime \prime}(t)=g(t), \quad-y^{\prime \prime}(t)=h(t) .
$$

Making use of the fact $k(0, s)=k(1, s)=0$ for $s \in[0,1]$, we obtain

$$
x(0)=y(0)=0 .
$$

Simple computations yield

$$
x(1)=\int_{0}^{1} y(t) d A(t), \quad y(1)=\int_{0}^{1} x(t) d B(t) .
$$

Hence, $(x, y) \in E \times E$ is a system of solutions of BVPs (2.3). 
Consider the linear differential system of BVPs

$$
\left\{\begin{array}{l}
-x^{\prime \prime}(t)=-M(t) x(t)+g(t), \quad-y^{\prime \prime}(t)=-N(t) y(t)+h(t), \quad t \in[0,1], \\
x(0)=y(0)=0, \quad x(1)=\int_{0}^{1} y(t) d A(t), \quad y(1)=\int_{0}^{1} x(t) d B(t),
\end{array}\right.
$$

where $M(t), N(t) \in C\left[[0,1], R^{+}\right]$and $g, h \in C[0,1]$.

Lemma 2.5 Assume that $\left(\mathrm{H}_{1}\right)$ holds. Then there exists a unique system of solutions $(x, y)$ to BVPs (2.8).

Proof It follows from Lemma 2.4 that (2.8) is equivalent to the operator equation

$$
(x, y)=T(x, y)+(\widetilde{g}, \widetilde{h}),
$$

where

$$
\begin{aligned}
& T(x, y)(t)=\left(-\int_{0}^{1} G_{1}(t, s) M(s) x(s) d s-\int_{0}^{1} H_{1}(t, s) N(s) y(s) d s,\right. \\
&\left.-\int_{0}^{1} G_{2}(t, s) N(s) y(s) d s-\int_{0}^{1} H_{2}(t, s) M(s) x(s) d s\right), \\
& \tilde{g}=\int_{0}^{1} G_{1}(t, s) g(s) d s+\int_{0}^{1} H_{1}(t, s) h(s) d s,
\end{aligned}
$$

and

$$
\widetilde{h}=\int_{0}^{1} G_{2}(t, s) h(s) d s+\int_{0}^{1} H_{2}(t, s) g(s) d s .
$$

By using standard arguments, we can easily show that $T: C[0,1] \times C[0,1] \rightarrow C[0,1] \times$ $C[0,1]$ is linear completely continuous. By Lemma 2.3 , the operator equation $(x, y)=$ $T(x, y)$ has only the zero solution. Then, by the Fredholm theorem, for given $(\widetilde{g}, \widetilde{h}) \in$ $C[0,1] \times C[0,1]$, the operator equation $(x, y)=T(x, y)+(\widetilde{g}, \tilde{h})$ has only one solution in $C[0,1] \times C[0,1]$. Hence, $(2.8)$ has exactly one system of solutions $(x, y) \in E \times E$.

\section{Main results}

In this section, on the basis of Lemma 2.2 and Lemma 2.5, using the monotone iterative technique, we shall show an existence theorem of a solution of (1.1).

We list the following assumptions for convenience.

$\left(\mathrm{H}_{2}\right) f_{1}(t, x, y)$ is nondecreasing in $y$ and there exists $M \in C\left[[0,1], R^{+}\right]$such that

$$
f_{1}\left(t, x_{1}, y\right)-f_{1}\left(t, x_{2}, y\right) \geq-M(t)\left(x_{1}-x_{2}\right)
$$

where $u_{0}(t) \leq x_{2} \leq x_{1} \leq \alpha_{0}(t), v_{0}(t) \leq y \leq \beta_{0}(t)$.

$\left(\mathrm{H}_{3}\right) f_{2}(t, x, y)$ is nondecreasing in $x$ and there exists $N \in C\left[[0,1], R^{+}\right]$such that

$$
f_{2}\left(t, x, y_{1}\right)-f_{2}\left(t, x, y_{2}\right) \geq-N(t)\left(y_{1}-y_{2}\right)
$$

where $u_{0}(t) \leq x \leq \alpha_{0}(t), v_{0}(t) \leq y_{2} \leq y_{1} \leq \beta_{0}(t)$. 
Theorem 3.1 Assume that $\left(u_{0}, v_{0}\right),\left(\alpha_{0}, \beta_{0}\right)$ are lower and upper systems of solutions of problem (1.1) such that $(2.1)$ holds, $f_{1}, f_{2} \in C\left[[0,1] \times R^{2}\right]$ and satisfies $\left(\mathrm{H}_{1}\right)-\left(\mathrm{H}_{3}\right)$. Then there exist monotone iterative sequences $\left\{\left(u_{n}, v_{n}\right)\right\},\left\{\left(\alpha_{n}, \beta_{n}\right)\right\}$ which converge uniformly on $[0,1]$ to the extremal solutions of problem (1.1) in the sector $\Omega$.

Proof $\forall(\xi, \eta) \in \Omega$, consider (2.8) with

$$
g(t)=f_{1}(t, \xi(t), \eta(t))+M(t) \xi(t), \quad h(t)=f_{2}(t, \xi(t), \eta(t))+N(t) \eta(t) .
$$

By Lemma 2.5, (2.8) has a unique system of solutions $(x, y) \in E \times E$. Denote an operator $S: \Omega \rightarrow E \times E$ by $(x, y)=S(\xi, \eta)$, and

$$
\begin{aligned}
S(\xi, \eta)(t)= & -\int_{0}^{1} G_{1}(t, s) M(s) x(s) d s-\int_{0}^{1} H_{1}(t, s) N(s) y(s) d s \\
& +\int_{0}^{1} G_{1}(t, s) N(s) g(s) d s+\int_{0}^{1} H_{1}(t, s) h(s) d s, \\
& -\int_{0}^{1} G_{2}(t, s) N(s) y(s) d s-\int_{0}^{1} H_{2}(t, s) M(s) x(s) d s \\
& \left.+\int_{0}^{1} G_{2}(t, s) h(s) d s+\int_{0}^{1} H_{2}(t, s) g(s) d s\right) .
\end{aligned}
$$

Then the operator $S$ has the following properties:

(i) $\left(u_{0}, v_{0}\right) \leq S\left(u_{0}, v_{0}\right), S\left(\alpha_{0}, \beta_{0}\right) \leq\left(\alpha_{0}, \beta_{0}\right)$.

Let $\left(u_{1}, v_{1}\right)=S\left(u_{0}, v_{0}\right), p(t)=u_{0}(t)-u_{1}(t)$ and $q(t)=v_{0}(t)-v_{1}(t)$. By $\left(\mathrm{H}_{2}\right)$ and $\left(\mathrm{H}_{3}\right)$, we have that

$$
\left\{\begin{array}{lc}
-p^{\prime \prime}(t) \leq-M(t) p(t), & -q^{\prime \prime}(t) \leq-N(t) q(t), \quad t \in(0,1), \\
p(0) \leq 0, \quad q(0) \leq 0, & p(1) \leq \int_{0}^{1} q(t) d A(t), \quad q(1) \leq \int_{0}^{1} p(t) d B(t),
\end{array}\right.
$$

which implies, by virtue of Lemma 2.2 , that $p(t) \leq 0, q(t) \leq 0, \forall t \in[0,1]$, i.e., $\left(u_{0}, v_{0}\right) \leq$ $S\left(u_{0}, v_{0}\right)$. A similar argument shows that $S\left(\alpha_{0}, \beta_{0}\right) \leq\left(\alpha_{0}, \beta_{0}\right)$. (ii) $S$ is nondecreasing. Let $\left(\xi_{1}, \eta_{1}\right),\left(\xi_{2}, \eta_{2}\right) \in \Omega$ be such that $\left(\xi_{1}, \eta_{1}\right) \leq\left(\xi_{2}, \eta_{2}\right)$. Suppose that $(p, q)=S\left(\xi_{1}, \eta_{1}\right)-S\left(\xi_{2}, \eta_{2}\right)$. By $\left(\mathrm{H}_{2}\right)$ and $\left(\mathrm{H}_{3}\right)$, we have

$$
\left\{\begin{array}{l}
-p^{\prime \prime}(t) \leq-M(t) p(t), \quad-q^{\prime \prime}(t) \leq-N(t) q(t), \quad t \in(0,1), \\
p(0)=q(0)=0, \quad p(1) \leq \int_{0}^{1} q(t) d A(t), \quad q(1) \leq \int_{0}^{1} p(t) d B(t),
\end{array}\right.
$$

which implies by virtue of Lemma 2.2 that $p(t) \leq 0, q(t) \leq 0, \forall t \in[0,1]$, i.e., $S$ is nondecreasing. This together with (i) implies that $S: \Omega \rightarrow \Omega$.

Now let $\left(u_{n}, v_{n}\right)=S\left(u_{n-1}, v_{n-1}\right),\left(\alpha_{n}, \beta_{n}\right)=S\left(\alpha_{n-1}, \beta_{n-1}\right), n=1,2,3, \ldots$. Following (i) and (ii), we have

$$
\begin{aligned}
\left(u_{0}, v_{0}\right) & \leq\left(u_{1}, v_{0}\right) \leq \cdots \leq\left(u_{n-1}, v_{n-1}\right) \leq\left(u_{n}, v_{n}\right) \leq \cdots \leq \cdots \\
& \leq\left(\alpha_{n}, \beta_{n}\right) \leq\left(\alpha_{n-1}, \beta_{n-1}\right) \leq \cdots \leq\left(\alpha_{1}, \beta_{1}\right) \leq\left(\alpha_{0}, \beta_{0}\right) .
\end{aligned}
$$


Using the standard arguments, it is easy to show that $\left\{\left(u_{n}, v_{n}\right)\right\}$ and $\left\{\left(\alpha_{n}, \beta_{n}\right)\right\}$ are uniformly bounded and equicontinuous in $\Omega$. By (3.1) and the Arzela-Ascoli theorem, we have

$$
\lim _{n \rightarrow \infty}\left(u_{n}, v_{n}\right)=\left(u_{*}, v_{*}\right), \quad \lim _{n \rightarrow \infty}\left(\alpha_{n}, \beta_{n}\right)=\left(\alpha^{*}, \beta^{*}\right),
$$

uniformly on $t \in[0,1]$, and $\left(u_{*}, v_{*}\right),\left(\alpha^{*}, \beta^{*}\right)$ satisfy (1.1). Moreover, $\left(u_{*}, v_{*}\right),\left(\alpha^{*}, \beta^{*}\right) \in \Omega$. Thus, $\left(u_{*}, v_{*}\right)$ and $\left(\alpha^{*}, \beta^{*}\right)$ are solutions of (1.1) in $\Omega$.

Next, we prove that $\left(u_{*}, v_{*}\right)$ and $\left(\alpha^{*}, \beta^{*}\right)$ are extremal solutions of (1.1) in $\Omega$. In fact, we assume that $(x, y)$ is any solution of (1.1). That is,

$$
\left\{\begin{array}{l}
-x^{\prime \prime}(t)=f_{1}(t, x(t), y(t)), \quad-y^{\prime \prime}(t)=f_{2}(t, x(t), y(t)), \quad t \in[0,1], \\
x(0)=y(0)=0, \quad x(1)=\int_{0}^{1} y(t) d A(t), \quad y(1)=\int_{0}^{1} x(t) d B(t) .
\end{array}\right.
$$

By $\left(\mathrm{H}_{2}\right)$ and $\left(\mathrm{H}_{3}\right)$, and Lemma 2.2, it is easy by induction to show that

$$
\left(u_{n}, v_{n}\right) \leq(x, y) \leq\left(\alpha_{n}, \beta_{n}\right), \quad n=1,2,3 \ldots
$$

Now, letting $n \rightarrow \infty$ in (3.2), we have $\left(u_{*}, v_{*}\right) \leq(x, y) \leq\left(\alpha^{*}, \beta^{*}\right)$. That is, $\left(u_{*}, v_{*}\right)$ and $\left(\alpha^{*}, \beta^{*}\right)$ are extremal systems of solutions of (1.1) in $\Omega$.

\section{Example}

Consider the following problems:

$$
\left\{\begin{array}{l}
-u^{\prime \prime}(t)=8\left(\frac{t}{2}-u(t)\right)^{3}+v^{3}(t), \quad t \in(0,1), \\
-v^{\prime \prime}(t)=t-2 v(t)+\arctan ^{3} u(t), \quad t \in(0,1), \\
u(0)=v(0)=0, \quad u(1)=2 v\left(\frac{1}{2}\right), \quad v(1)=\int_{0}^{1} u(t) d t,
\end{array}\right.
$$

where

$$
\alpha(t)=\left\{\begin{array}{ll}
0, & t \in\left[0, \frac{1}{2}\right), \\
2, & t \in\left[\frac{1}{2}, 1\right],
\end{array} \quad \beta(t)=t .\right.
$$

Obviously,

$$
\begin{gathered}
\kappa_{1}=1, \quad \kappa_{2}=\frac{1}{2}, \quad \kappa=\frac{1}{2}, \\
\left\{\begin{array}{l}
f_{1}(t, u, v)=8\left(\frac{t}{2}-u\right)^{3}+v^{3}, \\
f_{2}(t, u, v)=t-2 v+\arctan ^{3} u .
\end{array}\right.
\end{gathered}
$$

Take $\left(u_{0}(t), v_{0}(t)\right)=(0,0),\left(\alpha_{0}(t), \beta_{0}(t)\right)=(t, t)$, then

$$
\begin{aligned}
& \left\{\begin{array}{l}
-u_{0}^{\prime \prime}(t)=0 \leq t^{3}=8\left(\frac{t}{2}-u_{0}(t)\right)^{3}+v_{0}^{3}(t), \quad t \in(0,1), \\
-v_{0}^{\prime \prime}(t)=0 \leq t=t-2 v_{0}(t)+\arctan ^{3} u_{0}(t), \quad t \in(0,1), \\
u_{0}(0)=v_{0}(0)=0, \quad u_{0}(1)=0=2 v_{0}\left(\frac{1}{2}\right), \quad v_{0}(1)=0=\int_{0}^{1} u_{0}(t) d t,
\end{array}\right. \\
& \left\{\begin{array}{l}
-\alpha_{0}^{\prime \prime}(t)=0 \geq 0=8\left(\frac{t}{2}-\alpha_{0}(t)\right)^{3}+\beta_{0}^{3}(t), \quad t \in(0,1), \\
-\beta_{0}^{\prime \prime}(t)=0 \geq \arctan t-t \geq t-2 \beta_{0}(t)+\arctan ^{3} \alpha_{0}(t), \quad t \in(0,1), \\
\alpha_{0}(0)=\beta_{0}(0)=0, \quad \alpha_{0}(1)=1=2 \beta_{0}\left(\frac{1}{2}\right), \quad \beta_{0}(1)=1 \geq \frac{1}{2}=\int_{0}^{1} \alpha_{0}(t) d t .
\end{array}\right.
\end{aligned}
$$


It shows that $\left(u_{0}(t), v_{0}(t)\right)$ and $\left(\alpha_{0}(t), \beta_{0}(t)\right)$ are lower and upper systems of solutions of (4.1).

On the other hand, by (4.2), we have that

$$
\begin{aligned}
& f_{1}\left(t, x_{2}(t), y(t)\right)-f_{1}\left(t, x_{1}(t), y(t)\right) \geq-6 t^{2}\left(x_{2}(t)-x_{1}(t)\right), \\
& f_{2}\left(t, x(t), y_{2}(t)\right)-f_{2}\left(t, x(t), y_{1}(t)\right)=-2\left(y_{2}(t)-y_{1}(t)\right),
\end{aligned}
$$

where $u_{0}(t) \leq x_{1}(t) \leq x_{2}(t) \leq \alpha_{0}(t), v_{0}(t) \leq y_{1}(t) \leq y_{2}(t) \leq \beta_{0}(t)$.

Thus, all the conditions of Theorem 3.1 are satisfied. Therefore, by Theorem 3.1, (4.1) has an extremal system of solutions $\left(u_{*}, v_{*}\right),\left(\alpha^{*}, \beta^{*}\right)$, which can be obtained by taking limits from some iterative sequences.

\section{Competing interests}

The authors declare that they have no competing interests.

\section{Authors' contributions}

All authors typed, read and approved the final manuscript.

\section{Author details}

'School of Mathematical Sciences, Qufu Normal University, Qufu, Shandong 273165, P.R. China. ${ }^{2}$ Department of Mathematics, Shandong University of Science and Technology, Qingdao, 266590, P.R. China. ${ }^{3}$ Department of Statistics and Finance, Shandong University of Science and Technology, Qingdao, 266590, P.R. China.

\section{Acknowledgements}

The authors would like to thank the referees for carefully reading this article and making valuable comments and suggestions. The project is supported by the National Natural Science Foundation of China $(11371221,11071141)$, the Specialized Research Foundation for the Doctoral Program of Higher Education of China (20123705110001), the Program for Scientific Research Innovation Team in Colleges and Universities of Shandong Province, the Postdoctoral Science Foundation of Shandong Province and Foundation of SDUST.

\section{Received: 12 July 2013 Accepted: 18 October 2013 Published: 19 Nov 2013}

\section{References}

1. Aronson, DG: A comparison method for stability analysis of nonlinear parabolic problems. SIAM Rev. 20, 245-264 (1978)

2. Leung, A: A semilinear reaction-diffusion prey-predator system with nonlinear coupled boundary conditions: equilibrium and stability. Indiana Univ. Math. J. 31, 223-241 (1982)

3. Zettl, A: Sturm-Liouville Theory. Math. Surveys Monogr., vol. 121. Am. Math. Soc., Providence (2005)

4. Cabada, A: Extremal solutions and Green's functions of higher order periodic boundary value problems in time scales. J. Math. Anal. Appl. 290, 35-54 (2004)

5. Lakshmikanthan, V, Vatsala, AS: Basic theory of fractional differential equations. Nonlinear Anal. TMA 69, 2677-2682 (2008)

6. Nieto, JJ, Rodriguez-Lopez, R: Monotone method for first-order functional differential equations. Comput. Math. Appl. $52,471-484(2006)$

7. Nieto, JJ, Rodriguez-Lopez, R: Boundary value problems for a class of impulsive functional equations. Comput. Math. Appl. 55, 2715-2731 (2008)

8. Wei, Z, Li, Q, Che, J: Initial value problems for fractional differential equations involving Riemann-Liouville sequential fractional derivative. J. Math. Anal. Appl. 367, 260-272 (2010)

9. Zhang, S: Monotone iterative method for initial value problem involving Riemann-Liouville fractional derivatives. Nonlinear Anal. 71, 2087-2093 (2009)

10. Coster, CD, Habets, P: Two-Point Boundary Value Problems: Lower and Upper Solution. Elsevier, Amsterdam (2006)

11. Ladde, GS, Lakshmikantham, V, Vatsala, AS: Monotone Iterative Techniques for Nonlinear Differential Equations. Pitman, Boston (1985)

12. Zanolin, F: Nonlinear Analysis and Boundary Value Problems for Ordinary Differential Equations. Springer, New York (1996)

13. Asif, NA, Khan, RA: Positive solutions to singular system with four-point coupled boundary conditions. J. Math. Anal. Appl. 386, 848-861 (2012)

14. Protter, MH, Wiinberger, HF: Maximum Principles in Differential Equations. Prentice Hall International, Englewood Cliffs (1967) 